\title{
Prospects for poor-man's cloaking with low-contrast all-dielectric optical elements
}

\author{
Niels Asger Mortensen \\ asger@mailaps.org
}

Ole Sigmund

Olav Breinbjerg

\author{
Department of Photonics Engineering, Technical University of Denmark, DTU Fotonik, DTU-building \\ 345 west, DK-2800 Kongens Lyngby, Denmark \\ Department of Mechanical Engineering, Technical University of Denmark, Nils Koppels Allé, Building \\ 403, DK-2800 Kongens Lyngby, Denmark \\ Department of Electrical Engineering, Technical University of Denmark, Building 348, DK-2800 Kon- \\ gens Lyngby, Denmark
}

We discuss the prospects for low-contrast all-dielectric cloaking and offer a simple picture illustrating the basic obstacle for perfect cloaking without materials with an effective double-negative response. However, the same simple picture also gives directions for less perfect designs allowing for planar transmitted fields, but at the price of phase-slips which can only be eliminated at well-defined frequencies where the phase-slip amounts to a multiple of $2 \pi$. As a particular example, we consider assemblies of all-dielectric Lüneburg lenses forming a porous structure allowing for hiding objects inside the pores, independently on the polarization of the incident field. Cloaking must in general be realized with metamaterials realized through sub-wavelength structures, i.e. $\Lambda \ll \lambda$ with $\Lambda$ being the period and $\lambda$ the free-space wavelength. Interestingly, cloaking-like operations with Lüneburg-lens arrays perform in the opposite limit with $\Lambda \gg \lambda$. [DOI: $10.2971 /$ jeos.2009.09008]

Keywords: Lüneburg lens, cloaking

\section{INTRODUCTION}

The emergence of metamaterials and the demonstration of negative refraction [1] have revived the early ideas of Veselago [2] and the development of metamaterials is opening up for a variety of novel applications including cloaking [3, 4]. So far, cloaking devices have been employing metamaterials combining wires and split-ring resonators, but all-dielectric structures and the use of negative refraction in photonic crystals [5] have also been pursued [6]. While the design proposed by Pendry et al. is in principle capable of providing perfect cloaking, it has been shown how modifications and simplifications of the $\epsilon$ and $\mu$ profiles inevitably lead to imperfect cloaking $[7,8]$. Furthermore, material attenuation will naturally also work against the reconstructing of a transmitted field corresponding to the incident field in both phase and intensity. There is thus an interest in designing even less perfect cloaks both from an application point of view as well as for more fundamental studies. In this paper, we address the prospects for poor man's cloaking with the aid of low-contrast all-dielectric optical elements.

\section{PHASE-ADVANCE PICTURE}

The concept of cloaking involves the hiding of an object from interacting with an incident electromagnetic field, in the sense that the corresponding transmitted field remains unchanged with respect to both intensity and phase. The latter turns out to be the major challenge when it comes to low-contrast all-dielectric cloaking. While one may easily imagine an all- dielectric "cloaking" structure which causes a planar transmitted field, such a structure would inevitably be associated with phase slip between the field propagating in the "shadow" of the structure (red rays) and the field passing undisturbed by the structure (blue rays). This follows rigourously from the phase advance (given by the curve integral of the refractive index $n$ )

$$
\Delta \phi=k \int d \ell n(r)
$$

which for $n(\boldsymbol{r}) \geq 1$ will generally be different for the two classes of rays, except for particular values of the free-space wave vector $k=\omega / c=2 \pi / \lambda$ where the phase-slip equals an integer of $2 \pi$, thus resulting in a transmitted field with the desired completely planar phase front. We emphasize this as a particular simple picture illustrating why perfect cloaking appears impossible with low-contrast all-dielectric structures not employing dispersion effects of topological origin, such as photonic crystals supporting negative refraction [5]. Metamaterials on the other hand, provide $n<1$ and even $n<0$, thus allowing us to rewind the phase and thereby compensating for the physically longer optical path compared to the free-space rays not interacting with the structure. As we shall see, a lot of this trouble originates from the natural desire of having a cloak of a finite spatial extension.

However, relaxing the assumption of a structure with a finite extension in the direction transverse to the propagation of the incident light wave, one may actually perform cloakinglike operations with low-contrast all-dielectric structures. This would facilitate non-resonant quasi two-dimensional cloak- 


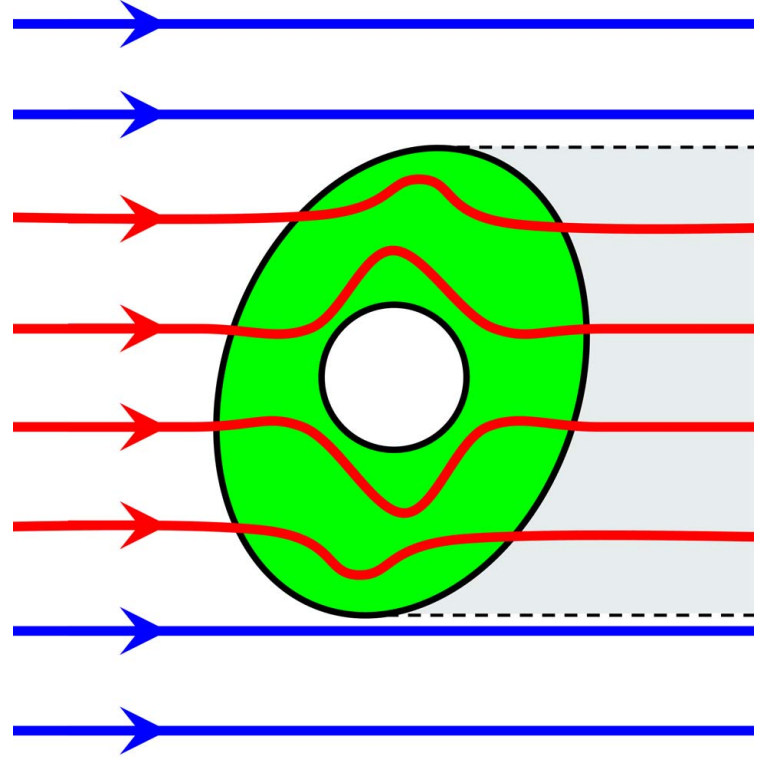

FIG. 1 Schematic illustration of a cloaking device. Rays are categorized according to those passing undisturbed by the cloak (blue) and rays traversing the cloak (red), but avoiding its hollow core. The "shadow" is indicated by grey, and dashed lines indicate the boundary across which a phase slip potentially occurs.

ing walls transparent to all wavelengths (limited by material losses), but still capable of supporting objects not visible to an observer in the far field.

\section{ARRANGEMENTS OF LÜNEBURG LENSES}

To explore the above idea we take advantage of the concept of inhomogeneous dielectric lenses with a long history, dating back to the 1940s with the work by Lüneburg (sometimes spelled Luneberg) published posthumous [9]. For more recent accounts, we refer to e.g. [10] and references therein. Today, such lenses are commercially available and finding widespread use for a variety of electromagnetic applications and especially in microwave communication and sensing technology. The work of Lüneburg has even inspired development of optical models for other physical phenomena, including more recent accounts for nuclear rainbow scattering [11]. Typically, the continuously varying graded-index profile of a Lüneburg lens is achieved with the aid of thin-layered structures, but more recently also photonic band-gap structures (or photonic crystals) have been exploited to mimic such index variations [12].

In a Lüneburg lens the graded index profile interpolates parabolically between the dielectric function of air $(\epsilon=1)$ at the outer radius $a$ to $\epsilon=2$ at the center of the lens, i.e.

$$
\epsilon_{L}(\boldsymbol{r})=n_{L}^{2}(\boldsymbol{r})=\left\{\begin{array}{cc}
2-\left(\frac{r}{a}\right)^{2}, & r<a, \\
1, & r>a .
\end{array}\right.
$$

For later convenience we have chosen a value of $\epsilon_{L}$ outside the lens corresponding to vacuum. The appealing thing about this graded-index profile is that for $\lambda \ll a$ it will focus an incident plane wave onto a single point on the opposite site of the lens, as may be checked by ray tracing, see panel (a) in Figure 2. The value of $\epsilon_{L}=1$ at the surface ensures impedance matching, thus avoiding back scattering, while the maximum value of $\epsilon_{L}=2$ causes all rays to have the same optical path length or phase advance, see Eq. (1), thus ensuring constructive interference at the opposite site of the lens. When the ray tracing assumption $\lambda \ll a$ is relaxed, diffraction phenomena modifies the focusing, but the overall picture in panel (a) in Figure 2 still applies, independently on the polarization of the incident field.

In the following we envision using the Lüneburg lens as a basic building block for more complex manipulation of plane wave fields. Panel (b) in Figure 2 illustrates one such example, where the lenses are arranged in a rectangular arrangement with a lattice constant $\Lambda=2 a$ so that one lens is touching its neighbors at the points where the rays focus. From ray tracing we immediately observe how the rays tend to avoid the pores of such a porous material. Potentially, one could use such electromagnetically "dead" regions (indicated by green) for cloaking. Contrary to the Pendry-type cloaks, the Lüneburg lens only requires dielectric materials with $\mu=1$ and $1<\epsilon<2$ which Nature is already providing to us ${ }^{1}$. Thus, no resonant phenomena are required and the frequency bandwidth will mainly be limited by material dispersion of the dielectric layers provided that $\lambda \ll \Lambda$. In most cases, material dispersion will not be a severe limitation. Furthermore, in terms of transparency the broad band width also comes to rescue since the Kramers-Kronig relations may put less strong bounds on this realization than it is the case for cloaking devices facilitated by strongly dispersive (and thereby lossy) metamaterial designs [13]. (a)

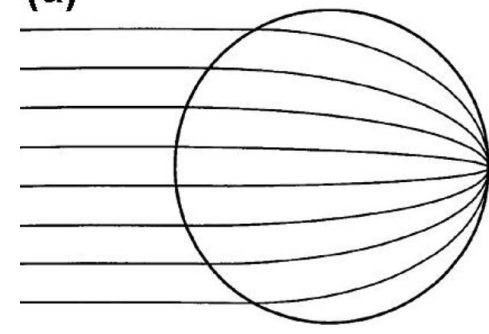

(b)

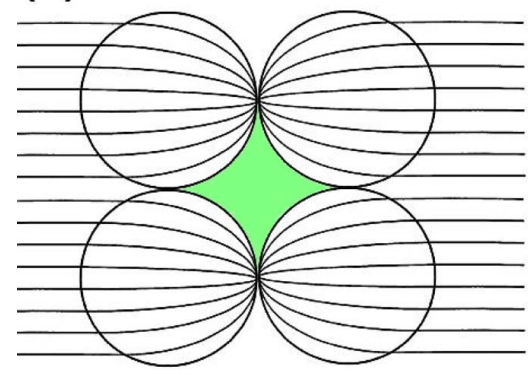

(c)

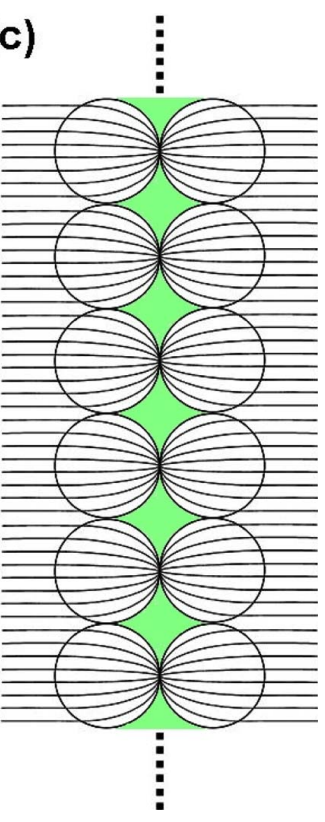

FIG. 2 Panel (a) shows the ray picture of a plane wave with $\lambda \ll a$ incident on a cylindrical Lüneburg lens of radius $a$. Panel (b) shows the ray picture in the case of 4 lenses arranged in a two-by-two quadratic arrangement with a lattice constant $\Lambda=2 a$. Panel (c) shows the the ray picture of structure repeated periodically in the vertical direction. Note how no rays are supported in pore regions of the lens arrays (green regions) in panels (b) and (c).

\footnotetext{
${ }^{1}$ In an experiment one may take advantage of index-matching fluids with $\epsilon_{f}$ in which case Eq. (4) should be changed accordingly to $\epsilon_{L} \rightarrow \epsilon_{L} \times \epsilon_{f}$.
} 

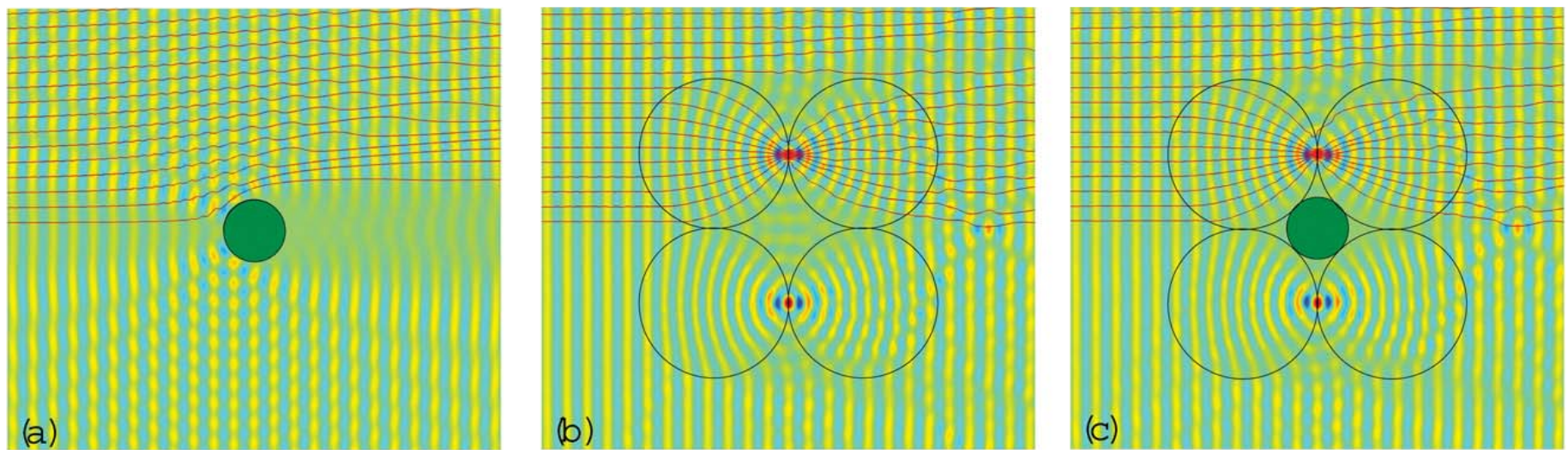

FIG. 3 (Finite-element simulation of Eq. (3), showing the wave interaction with a poor man's cloak formed by a two-by-two array of Lüneburg lenses in the case of $\lambda / a=3.679$. Panel (a) shows how an isolated high-index dielectric object (rod with $\epsilon=1000$ and radius $b / a=\sqrt{2}-1$ ) scatters an incident plane wave. Panel (b) shows the corresponding results for the "empty", while panel (c) shows the result when the scattering object in panel (a) is placed inside the cloak.

\section{RAYTRACING VERSUS WAVE SIMULATIONS}

To further illustrate the cloaking perspectives and support the ray tracing picture, we next solve the Maxwell equations numerically, thus also accounting for possible diffraction effects. The full wave dynamics is governed by a wave equation which for the electrical field takes the following form

$$
\nabla \times \nabla \times E(r)=k^{2} \epsilon(\boldsymbol{r}) \boldsymbol{E}(\boldsymbol{r})
$$

Generalizing to an arrangement of several Lüneburg lenses, see e.g. panel (b) in Figure 2, we have a spatially varying dielectric function given by

$$
\epsilon(\boldsymbol{r})=1-N+\sum_{j=1}^{N} \epsilon_{L}\left(\boldsymbol{r}-\boldsymbol{r}_{j}\right)
$$

with center positions of the $N$ non-overlapping lenses given by $\boldsymbol{r}_{j}$. In Figure 3 we consider a two-by-two array of Lüneburg lenses. To fully appreciate the potential of this structure we show finite-element simulations for cloaking of a strongly scattering object. Panel (a) shows how an isolated high-index dielectric object strongly scatters an incident plane wave from the left. Panel (b) shows similar results for the "empty" twoby-two lens array, transforming the incident plane wave into a transmitted plane wave. Finally, panel (c) shows the corresponding result when the scattering object in panel (a) is placed inside the cloak. We emphasize how the transmitted fields in panels (b) and (c) are very similar and at the same time very different from the far field of the strongly scattering object itself, see panel (a). However, we emphasize that while the transmitted field to the right of the device is seen to have a plane phase front independently of the cloak being empty or occupied by a dielectric rod, we also observe a phase slip at the vertical distance where the incident ray is tangential to the surface of the lens. Denoting the vertical direction (perpendicular to the free-space rays) by $y$, we have

$$
\Delta \phi(|y| a)-\Delta \phi(|y| a)=(\pi-2) k a
$$

and thus it will only be for the following values of the wave vector,

$$
k_{m} a=m \frac{2 \pi}{\pi-2}
$$

with $m$ being an integer, that the transmitted field is a perfect reconstruction in terms of both phase and intensity, i.e. a full recovery of the wave front. The propagation of the incident field into a perfect planar transmitted field, in terms of both intensity and phase, will thus only occur for distinct frequencies. However, we emphasize that since the double-negative response of any metamaterial will also have a resonant origin, the above requirement, Eq. (6), is perhaps not a too severe limitation compared to the performance of practical realizations of Pendry-type cloaking devices [4]. The fact that the present structure works perfectly at a series of discrete frequencies, Eq. (6), could be an advantage over metamaterial based cloaks where frequency tuning is pursued to cover more frequencies [14].

\section{PERIODIC ARRANGEMENTS}

Having considered the configuration of a finite number of Lüneburg lenses we extend the concept to a situation where the structure is repeated in a periodic way in, say, the $y$ direction, while still having a finite extension in the $x$ direction, see panel (c) in Figure 2. Rather than acting like a carpet-like cover, such a structure would for normally incident light behave like a porous transparent carpet or blanket even with its pores infiltrated by strongly non-transparent objects or substances. For such a blanket, the transmitted field would be planar and the phase-slip discussed above, see Eq. (5), would only be an edge phenomenon. In the near-field region where the above discussion of the phase-slip is unimportant, the device will thus basically work for any frequency. Finally, it is noted that to maintain the correct order of the rays as they traverse the cloaking structure, and thus ensure a distortion-free transmitted field, a multiple of four layers of lenses is actually necessary.

So far we have only addressed the very particular case of an incoming plane normal to the plane of the array of Lüneburg lenses, i.e. $\theta=0$. A deviation from normal incidence is expected to change the above behavior since the focal point will then no longer coincide with the point of contact between the neighboring spheres. As a consequence, the flow of energy between the spheres will then start to occur also in the originally 
field-free void regions, i.e. the spaces where the cloaking were supposed to occur! We explore this in more detail, by considering the time-averaged power flow $\langle S\rangle_{t}$, evaluated on the right-hand side of the array. For simplicity, we use the following dimensionless error measure

$$
\gamma(\theta) \equiv \frac{\int d y \hat{\boldsymbol{x}} \times\left\langle\boldsymbol{S}(\theta)-\boldsymbol{S}_{0}\right\rangle_{t}}{\int d y \hat{\boldsymbol{x}} \times\left\langle\boldsymbol{S}_{0}\right\rangle_{t}}
$$

to monitor how a finite angle of incidence $\theta$ causes deviations from a transmitted perfect plane wave. Here, $\left\langle S_{0}\right\rangle_{t}$ is the timeaveraged Poynting vector of the incident plane wave, so that formally $\gamma=0$ in the absence of both the lens array and the obstacle while it is finite in the presence of the lens array alone or the lens array containing an obstacle. Figure 4 shows $\gamma$ as a function of $\theta$ in the case where $\lambda / a=3.774$ for a structure repeated in the vertical direction, corresponding to panel (c) in Figure 2. As expected, we observe an overall increase in $\gamma$ when the plane wave has a finite angle of incidence. However, interestingly we also observe distinct minima at particular values of the angle. For the particular choice of frequency the minimum occur for $\theta$ around 9 degrees. Field plots (not shown) indicate that the minimum is related to a negative refraction of the incident planewave at these particular angles, but this quite interesting phenomena will be reported elsewhere [15]. For significant deviations from normal incidence, the evident angle sensitivity should be considered and it may eventually represent a major obstacle for the practical use of the lens array.

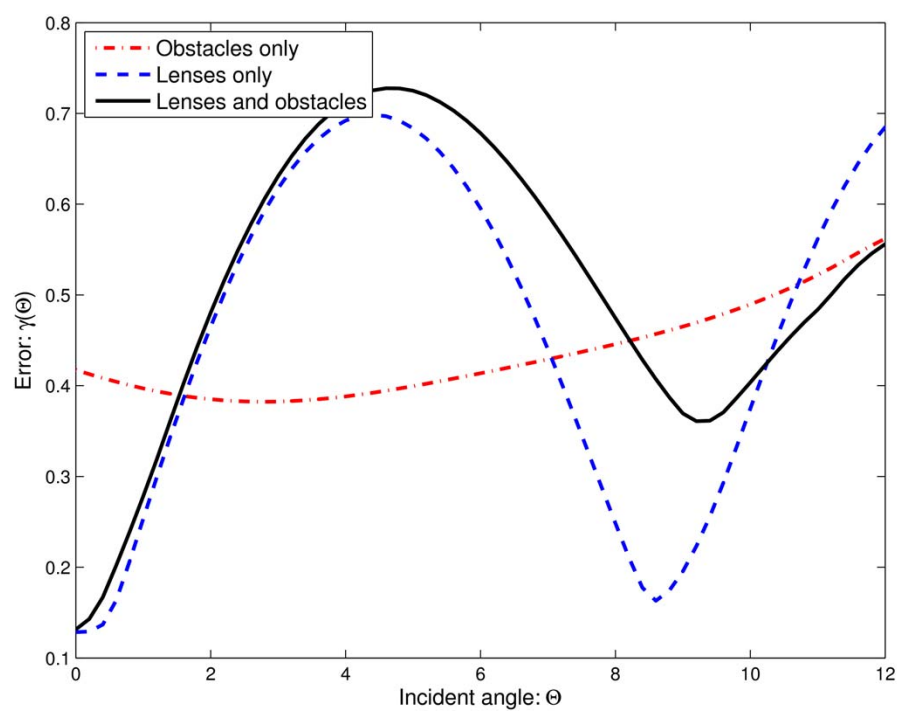

FIG. 4 Plot of the error measure $\gamma$ versus angle of incidence, see Eq. (7), for a structure corresponding to panel (c) in Figure 2 with a wavelength of $\lambda=a / 3.774$.

\section{CONCLUSION}

In conclusion, while perfect cloaking puts strong demands on the material constitutive parameters and calls for doublenegative metamaterials, we have here shown how cloakinglike phenomena may be explored with much simpler alldielectric graded-index materials. As an example, we have shown how a simple arrangement of Lüneburg lenses may be used to hide objects in a plane-wave incident field, independently of its polarization. Such graded index materials are readily available and the proposed concept could find use in applications seeking to screen objects from interacting with incident electromagnetic radiation, while still leaving the system fully transparent for the electromagnetic radiation. While the proposed structure obviously only works for near normal incidence, the clear advantage over complicated metamaterial approaches, employing true sub-wavelength resonating structures, is that Luneburg lenses are simple graded-index structures already commercially available for a range of frequency regimes with high quality and transparency. Finally, acoustic cloaking has recently been proposed $[16,17]$ and we believe that the concepts we propose here could be extended to mechanical structures with graded mass density, to the benefit of also acoustical applications.

We have for simplicity emphasized two-dimensional structures (or equivalently two-dimensional arrays of cylinders), but the generalization to three-dimensional structures realized through stacking of spherical Lüneburg lenses may seem obvious. However, it should be emphasized that not all of the pores are useful for hiding objects. This is perhaps best appreciated by considering the cubic two-by-two-by-two arrangement lenses, where rays having a normal incidence of one of the faces will still pass through the center of the structure.

\section{ACKNOWLEDGMENTS}

This work is supported by the Danish Research Council for Technology and Production Sciences (grant no: 274-06-0507).

\section{References}

[1] J. B. Pendry and D. R. Smith, "Reversing light with negative refraction" Phys. Today 57, 37-43 (2004).

[2] V. G. Veselago, "Electrodynamics of substances with simulataneously negative values of $\epsilon$ and $\mu$." Sov. Phys. Uspekhi-USSR 10, 509 (1968).

[3] J. B. Pendry, D. Schurig, and D. R. Smith, "Controlling electromagnetic fields" Science 312, 1780 - 1782 (2006).

[4] D. Schurig, J. J. Mock, B. J. Justice, S. A. Cummer, J. B. Pendry, A. F. Starr, and D. R. Smith, "Metamaterial electromagnetic cloak at microwave frequencies" Science 314, 977-980 (2006).

[5] M. Notomi, "Theory of light propagation in strongly modulated photonic crystals: Refractionlike behavior in the vicinity of the photonic band gap" Phys. Rev. B 62, 10696-10705 (2000).

[6] D. P. Gaillot, C. Croenne, and D. Lippens, "An all-dielectric route for terahertz cloaking" Opt. Express 16, 3986-3992 (2008).

[7] Z. Ruan, M. Yan, C. W. Neff, and M. Qiu, "Ideal cylindrical cloak: Perfect but sensitive to tiny perturbations" Phys. Rev. Lett. 99, 113903 (2007).

[8] M. Yan, Z. C. Ruan, and M. Qiu, “Cylindrical invisibility cloak with simplified material parameters is inherently visible" Phys. Rev. Lett. 99, 233901 (2007).

[9] R. K. Lüneburg, Mathematical Theory of Optics (University of California Press, Berkeley, 1964).

[10] A. D. Greenwood and J. M. Jin, "A field picture of wave propagation in inhomogeneous dielectric lenses" IEEE Antennas Propag. 41, 918 (1999). 
[11] F. Michel, G. Reidemeister, and S. Ohkubo, "Luneburg lens approach to nuclear rainbow scattering" Phys. Rev. Lett. 89, 152701 (2002).

[12] Y. J. Park, A. Herschlein, and W. Wiesbeck, "A photonic bandgap (PBC) structure for guiding and suppressing surface waves in millimeter-wave antennas" IEEE T. Microw. Theory 49, 1854-1859 (2001).

[13] M. I. Stockman, “Criterion for negative refraction with low optical losses from a fundamental principle of causality" Phys. Rev. Lett. 98, 177404 (2007).

[14] D. X. Wang, H. S. Chen, L. X. Ran, J. T. Huangfu, J. A. Kong, and
B. L. Wu, "Reconfigurable cloak for multiple operating frequencies" Appl. Phys. Lett. 93, 043515 (2008).

[15] 0. Sigmund, N. A. Mortensen, and 0. Breinbjerg (unpublished, 2008).

[16] G. W. Milton, M. Briane, and J. R. Willis, “On cloaking for elasticity and physical equations with a transformation invariant form" New J. Phys. 8, 248 (2006).

[17] A. N. Norris, "Acoustic cloaking theory" Proc. R. Soc. A. Eng. Sci. 464, 2411-2434 (2008). 